AperTO - Archivio Istituzionale Open Access dell'Università di Torino

\title{
Comparison between cluster and supercell approaches: the case of defects in diamond
}

\section{This is the author's manuscript}

Original Citation:

Availability:

This version is available http://hdl.handle.net/2318/1640619

since 2017-05-30T17:40:32Z

Published version:

DOI:10.1007/s00214-017-2071-5

Terms of use:

Open Access

Anyone can freely access the full text of works made available as "Open Access". Works made available under a Creative Commons license can be used according to the terms and conditions of said license. Use of all other works requires consent of the right holder (author or publisher) if not exempted from copyright protection by the applicable law. 


\title{
Comparison Between Cluster and Supercell Approaches: The Case of Defects in Diamond
}

\author{
Simone Salustro · Anna Maria Ferrari* · Roberto Orlando · Roberto Dovesi
}

Received: date / Accepted: date

\begin{abstract}
The results produced by the cluster and the supercell approaches, when applied to the study of the vacancy and $\langle 100\rangle$ split self-interstitial defects in diamond, are critically compared. The same computer code, CRYSTAL, basis set and DFT functional (the hybrid B3LYP) are used. Clusters of increasing size (from 35 to $969 \mathrm{C}$ atoms) are considered, and the results compared to those from a supercell containg $128 \pm 1$ atoms, for which the interaction between defects in different cells can be considered negligible. It is shown that geometry and energy data (atomic relaxation, defect formation energy, relative energy between different spin states) show a very local nature and then converge rapidly with the cluster size. Other properties, frequently used for the characterization of the defects using relatively small clusters (band gaps, impurity energy levels in the gap, Raman spectra) converge slowly, and also at the limit of the very large clusters here considered, still differ from the periodic counterpart.
\end{abstract}

\section{S. Salustro}

Dipartimento di Chimica, Università di Torino, via Giuria 5, 10125 Torino, Italy.

\section{*A. M. Ferrari}

Dipartimento di Chimica and NIS (Nanostructured Interfaces and Surfaces) Centre, Università di Torino, via Giuria 5, 10125 Torino, Italy. E-mail: anna.ferrari@unito.it

\section{R. Orlando}

Dipartimento di Chimica and NIS (Nanostructured Interfaces and Surfaces) Centre, Università di Torino, via Giuria 5, 10125 Torino, Italy.

R. Dovesi

Dipartimento di Chimica and NIS (Nanostructured Interfaces and Surfaces) Centre, Università di Torino, via Giuria 5, 10125 Torino, Italy.

\section{Introduction}

The cluster approach is widely used to study local defects [1-10], chemisorption at crystalline surfaces [11-16], molecular crystals [17-19] and, in extreme cases, the properties of bulk solids [20,21]. A particular advantage of this method, in which the infinite system is approximated by a finite cluster containing a relatively small number of atoms, is that sophisticated many-body techniques of quantum chemistry are available that can be used to estimate electron correlation effects[22,23] and for the characterization of excited states. [24-26] There are however also disadvantages as compared to the supercell scheme [27-30], the most important of which is the spurious perturbation arising from the abrupt truncation of the infinite lattice. For ionic systems, the dominant contribution from the missing ions is the Coulomb field, which can be approximated through the introduction of a finite set of charges [10-12,16,31,32] or by applying more sophisticated (and numerically delicate) embedding schemes.[3,33] Bare clusters have been often used for mixed ionic-covalent oxides as for modeling $\mathrm{TiO}_{2}$. [25, 26,34] In metals and covalent solids, by contrast, border quantum effects are important and not easily simulated. In the latter case the atoms at the surface of the cluster would normally be bound to other atoms and, to simulate these bonds whilst retaining the possibility of working with a finite cluster, the usual approach is to saturate the "dangling bonds" with hydrogen atoms. [13-15,35,36] A number of ambiguities remains, however, such as the importance of overlap terms between the cluster and the missing infinite crystal (a quantum effect), the field modification arising from the presence of the hydrogen atoms and the appropriate method to use in the case of mixed ionic-covalent situations.

A justification of the cluster models is often looked for $a$ posteriori in the inner consistency of the method, i.e. convergence of results with cluster size, and comparison with 
other calculations or experimental data. However, convergence with cluster size can be very slow, and tend to the wrong limit (the surface perturbation increases with cluster size), as we will show for the band gap. Furthermore, the cost of such calculations increases quickly, so in most of the cases the largest clusters considered contain rather a small number of atoms (say less than 100-200).[6,7] Comparisons with periodic or embedded clusters performed in the past $[6,37,38]$ can be considered only qualitative, since differences in functionals, basis set, computational parameters and adopted computer code can introduce severe differences in the results.

The cluster approach is particularly favorable for a study of defects in semiconductors, since long range electrostatic and relaxation effects are expected to be negligible. For this reason the cluster model has been extensively used also in recent years for the study of local defects in diamond, and actually the most complete investigations have been performed with this technique.[6-9]

However, a clear evidence of the convergence with the cluster size of the various properties has not been provided. In some cases, when both supercell and cluster schemes have been used in the same investigation, different functionals have been used for the two cases, making meaningless the quantitative comparison of the results.[6] Finally, when properties that can hardly be defined as local, as the IR or Raman vibrational spectrum, have been computed, border effects were not discussed. More explicitly: how border effects perturb the very simple Raman spectrum of pristine diamond, containing a single peak at $1332 \mathrm{~cm}^{-1}$ ? And when new peaks appear in the spectrum, can they be attributed to the defect or to border effects?

In this work the cases of the neutral vacancy and the $\langle 100\rangle$ split self-interstitial defects in diamond are considered. Defect formation energies, atomic relaxations, band structure of the defect, relative energies of different spin states and Raman spectra are investigated as a function of the cluster size, and are compared directly with the results of wellconverged supercells calculations.[39-41]. This latter method involves a periodic array of unit cells of the host crystal containing the defect at the centre. If the cell is sufficiently large, interactions between the defects belonging to different cells is negligible; it was shown in a previous paper [42] that a 128 atom supercell is large enough to accurately describe the defects we are going to discuss here. Both supercell and cluster calculations were performed using the same computer code, namely CRYSTAL [43], basis set and DFT functional. In this way, differences between the results of the two methods may be attributed completely to the model (cluster or periodic boundary conditions).

The structure of the paper is as follows: in Section 2 the studied diamond clusters are presented together with the computational methods adopted for their simulation. In Section 3 results obtained on pristine and defective clusters are presented. Finally, in Section 4 conclusions are drawn.

\section{Models and Methods}

\subsection{Cluster}

Clusters have been built following a scheme (performed automatically by the CLUSTER option of the CRYSTAL code $[43,44])$ according to which, starting from the central atom, the connectivity along the covalent bonds is followed, in a way that: i) the 24 symmetry operators of the $T_{d}$ group are all maintained, ii) a given number of shells of neighbors is included in the cluster definition, iii) the cluster is terminated when the surface atoms present a minimal set of unsaturated bonds; this guarantees the largest possible $\mathrm{C} / \mathrm{H}$ ratio $\mathrm{R}$, and then the smallest effect of the surface of the cluster. Saturating hydrogen atoms are placed along the $\mathrm{C}-\mathrm{C}$ directions, at $1.10 \AA$ from the $\mathrm{C}$ atom. Several clusters of increasing size (including from 2 up to 32 shells of neighbors) have been selected: $\mathrm{C}_{35} \mathrm{H}_{36}(\mathrm{R}=0.97), \mathrm{C}_{71} \mathrm{H}_{60}$ $(\mathrm{R}=1.18), \mathrm{C}_{165} \mathrm{H}_{108}(\mathrm{R}=1.65), \mathrm{C}_{265} \mathrm{H}_{140}(\mathrm{R}=1.89), \mathrm{C}_{455} \mathrm{H}_{196}$, $(\mathrm{R}=2.32), \mathrm{C}_{651} \mathrm{H}_{252}(\mathrm{R}=2.58)$ and $\mathrm{C}_{969} \mathrm{H}_{324}(\mathrm{R}=2.99)$, see Figure 1 and Table 1. Our scheme is similar to the one adopted by Beuer and Briddon and by Kertesz and coworkers for their studies on pristine and defective diamond (see for instance Fig. 2 of Refs.[7,6]). The vacancy defect has been created through the substitution of the central atom with a ghost atom (see next paragraph). In the same way the interstitial defect has been created by adding a $\mathrm{C}$ atom near the central one. The corresponding cluster has been denominated specifying that one $\mathrm{C}$ atom is missing or has been added: then, for instance, $\mathrm{C}_{35-1} \mathrm{H}_{36}$ and $\mathrm{C}_{35+1} \mathrm{H}_{36}$ indicate, respectively, a cluster with a vacancy or an interstitial defect. Cluster properties have been compared with those of supercells $\left(\mathrm{S}_{128}\right)$ containing, before the defect creation, 128 carbon atoms [39-41].

\subsection{Computational details}

All calculations have been performed by employing the $a b$ initio CRYSTAL code[43], the B3LYP "hybrid" functional[45, 46] and a split valence 6-21G basis set[47] for the carbon atoms, with exponent of the most diffuse Gaussian optimized for the non defective periodic structure $\left(\alpha=0.228 \mathrm{Bohr}^{-2}\right)$. This combination has shown to provide accurate description of the properties of both regular and defective diamond. [39-41] In the cluster calculations, terminal hydrogen atoms have been described at the STO-3G[48] level. To increase the variational freedom in the vacancy region, the same $\mathrm{C}$ basis set has been placed at the vacancy site (see the GHOSTS option in the CRYSTAL manual[44]). 

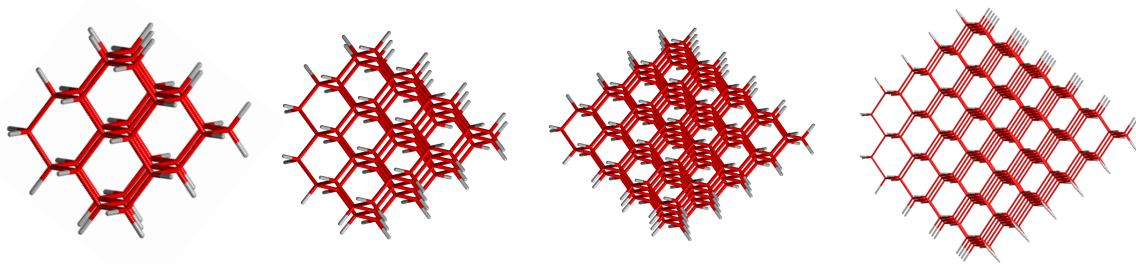

$$
\mathrm{C}_{35} \mathrm{H}_{36}
$$

$$
\mathbf{C}_{71} \mathbf{H}_{60}
$$

$$
\mathrm{C}_{165} \mathrm{H}_{100}
$$

$$
\mathrm{C}_{265} \mathrm{H}_{140}
$$
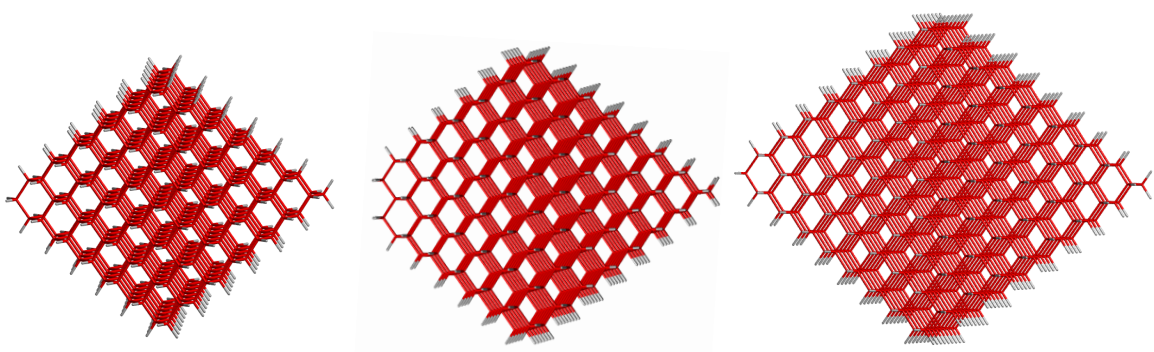

$\mathrm{C}_{455} \mathrm{H}_{196}$

$\mathrm{C}_{651} \mathrm{H}_{252}$

$\mathrm{C}_{969} \mathrm{H}_{324}$

Fig. 1 The six diamond clusters considered in this study. The carbon backbone is reported in red while terminal hydrogen atoms are in white.

Table 1 Structure and Mulliken charges $(q)$ of pure diamond clusters. Roman numerals label the stars of neighbors of the center of the cluster, defined in terms of the distance from the center; stars VI and VII are "degenerate", as they contain sets of atoms not symmetry related. $n$ is the

\begin{tabular}{|c|c|c|c|c|c|c|c|c|c|}
\hline \multirow{2}{*}{\multicolumn{2}{|c|}{$\begin{array}{l}\text { Shell of neighbors } \\
\mathrm{d}(\AA)\end{array}$}} & 0 & I & II & III & IV & V & VI & VII \\
\hline & & 0.0 & 1.56 & 2.55 & 2.99 & 3.61 & 3.93 & 4.42 & 4.68 \\
\hline \multirow{5}{*}{$n(h)$} & $\mathrm{C}_{35} \mathrm{H}_{36}$ & $1(0)$ & $4(0)$ & $12(1)$ & $12(1)$ & $6(2)$ & - & - & - \\
\hline & $\mathrm{C}_{71} \mathrm{H}_{60}$ & $1(0)$ & $4(0)$ & $12(0)$ & $12(1)$ & $6(0)$ & $12(1)$ & $12(2)$ & - \\
\hline & $\mathrm{C}_{165} \mathrm{H}_{100}$ & $1(0)$ & $4(0)$ & $12(0)$ & $12(0)$ & $6(0)$ & $12(0)$ & $24(0)$ & $16(1)$ \\
\hline & $\mathrm{C}_{455} \mathrm{H}_{196}$ & $1(0)$ & $4(0)$ & $12(0)$ & $12(0)$ & $6(0)$ & $12(0)$ & $24(0)$ & $16(0)$ \\
\hline & $\mathrm{C}_{651} \mathrm{H}_{252}$ & $1(0)$ & $4(0)$ & $12(0)$ & $12(0)$ & $6(0)$ & $12(0)$ & $24(0)$ & $16(0)$ \\
\hline \multirow{5}{*}{$q$} & $\mathrm{C}_{35} \mathrm{H}_{36}$ & -0.027 & -0.065 & 0.075 & 0.062 & 0.197 & - & - & - \\
\hline & $\mathrm{C}_{71} \mathrm{H}_{60}$ & -0.018 & -0.014 & -0.055 & 0.106 & -0.087 & 0.077 & 0.207 & - \\
\hline & $\mathrm{C}_{165} \mathrm{H}_{100}$ & 0.000 & -0.004 & -0.008 & -0.012 & -0.014 & -0.050 & $(0.030)$ & $(-0.015)$ \\
\hline & $\mathrm{C}_{455} \mathrm{H}_{196}$ & 0.000 & 0.000 & 0.000 & 0.000 & 0.000 & -0.005 & -0.004 & -0.004 \\
\hline & $\mathrm{C}_{651} \mathrm{H}_{252}$ & 0.000 & 0.000 & 0.000 & 0.000 & 0.000 & 0.000 & 0.000 & 0.000 \\
\hline
\end{tabular}
total number of $\mathrm{C}$ atoms in a given shell of neighbors, while $(h)$ is the number of $\mathrm{H}$ atoms in the coordination sphere of each carbon in the shell. $\mathrm{C}_{455} \mathrm{H}_{196}$ has 32 stars of neighbors, the $\mathrm{C}$ atoms of 15 of them (up to a distance of $6.92 \AA$ ) are linked to four $\mathrm{C}$ atoms; the radius of the cluster is

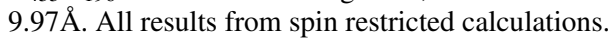

The level of accuracy in evaluating the Coulomb and HartreeFock exchange series is controlled by five parameters $\left(\mathrm{T}_{i}\right)[44]$ that were set to $\mathrm{T}_{1}-\mathrm{T}_{4}=8$ and $\mathrm{T}_{5}=16$ (see also Refs. [49, 50]). The convergence threshold for the self-consistent field (SCF) calculations was set to $10^{-8}$ Hartree for the total energy and to $10^{-10}$ hartree for vibration frequency calculations; default thresholds[44] have been used for the geometry optimizations. Cluster SCF equations have been solved at the $\Gamma$ point of the reciprocal lattice while $4 \mathbf{k}$-points in the irreducible part of the Brillouin zone (corresponding to a "shrinking factor" of 4) had been selected for the reference $\mathrm{S}_{128}$ supercell calculations, see Refs. [39-41] for details. In geometry optimization two different procedure have been followed: in the former only the $\mathrm{H}$ atoms have been kept fixed at their initial positions and all $\mathrm{C}$ atoms have been fully relaxed (we refer to these clusters as FULL); in the latter approach only $\mathrm{C}$ atoms in the internal shells of the clusters have been relaxed, that means all $\mathrm{C}$ atoms not linked to $\mathrm{H}$ but also not at the border of the cluster (we refer to these clusters as FRAGMENT). The FRAGMENT option has been applied only to selected clusters. Therefore, if not otherwise specified, when discussing properties of clusters we refer to the fully relaxed ones.

The presence of defects in diamonds generates unpaired electrons. The corresponding magnetic states are identified by the $S_{z}$ value (the sum of alpha and beta spins of the elec- 
trons in the unit cell); $\mathrm{S}_{z}$ is related to the spin multiplicity $M$ as follows: $M=2 S_{z}+1$. In order to calculate the correct spin state an appropriate guess is selected by setting the atomic spins accordingly (see the ATOMSPIN option in the CRYSTAL manual[44]). A few additional options are available for driving the SCF process towards a well converged solution. One of these options is SPINLOCK that locks the spin state for a given number of SCF cycles. Another is GUESSP, that starts the present case from the density matrix of a similar case. Spin restricted calculations are used for non defective diamond whereas unrestricted spin calculations for the defective systems.

Harmonic phonon frequencies (i.e. wave-numbers), $\omega_{p}$, at the $\Gamma$ point (i.e. at the center of the first Brillouin zone in reciprocal space) are obtained from the diagonalization of the mass-weighted Hessian matrix of the second energy derivatives with respect to atomic displacements $u$ :[51-55]

$W_{a i, b j}^{\Gamma}=\frac{H_{a i, b j}^{\mathbf{0}}}{\sqrt{M_{a} M_{b}}} \quad$ with $\quad H_{a i, b j}^{\mathbf{0}}=\left(\frac{\partial^{2} E}{\partial u_{a i}^{\mathbf{0}} \partial u_{b j}^{\mathbf{0}}}\right)$,

where atoms $a$ and $b$ (with atomic masses $M_{a}$ and $M_{b}$ ) in the reference cell, $\mathbf{0}$, are displaced along the $i$-th and $j$-th Cartesian directions, respectively. For finite systems the scheme is exactly the same.

The Raman intensity of the Stokes line of a phonon mode $Q_{p}$, characterized by a frequency $\omega_{p}$, active due to the $\alpha_{i i^{\prime}}$ component of the polarizability tensor $\alpha$, is given by:

$I_{i i^{\prime}}^{p} \propto\left(\frac{\partial \alpha_{i i^{\prime}}}{\partial Q_{p}}\right)^{2}$.

The relative Raman intensities of the peaks are computed analytically by exploiting a scheme, implemented in the CRYSTAL14 program, $[56,57]$ which constitutes an extension of the analytical calculation of infrared (IR) intensities. [57,58] Both schemes are based on the solutions of first- and secondorder Coupled-Perturbed- Hartree-Fock/Kohn-Sham equations.[59,60] The Raman spectrum is then computed by considering the transverse-optical (TO) modes and by adopting a pseudo-Voigt functional form: a linear combination of a Lorentzian and a Gaussian curve with full width at half maximum of $6 \mathrm{~cm}^{-1}$ are adopted. Raman intensities are normalized so that the highest value is conventionally set to 1000 arbitrary units.

\section{Results}

\subsection{Non defective diamond}

As a preliminary step, we consider the case of the non defective system. For selected but relevant properties we will try to verify how deeply the border perturbation propagates inwards. In Table 1 the structure and Mulliken net charges (up to the VII shell of neighbors, when available, of the atom at the origin) of five pure diamond clusters are reported.

The Mulliken net charges $(q)$ that should be zero in the perfect bulk, can be used as a measure of the penetration of surface effects toward the center of the cluster. Since hydrogen is slightly less electronegative than carbon (2.20 and 2.55, respectively, according to Pauling),[61] it leaves a fraction of an electron to the $\mathrm{C}$ atom to which is linked, and a sort of charge wave propagates inwards. In $\mathrm{C}_{35} \mathrm{H}_{36}$ the net charge on first neighbors is still as large as $-0.065|e|$ per atom (overall $-0.26|e|$ in the star of four neighbors, far from negligible), while for $\mathrm{C}_{71} \mathrm{H}_{60}$ net charges are smaller by about a factor two; nevertheless, from the third shell of neighbors, relatively large positive-negative charge oscillations take place. It is only in $\mathrm{C}_{165} \mathrm{H}_{100}$ that the central atom of the cluster has a negligible net charge (less than $0.005|e|$ in module), and four stars of neighbors with Mulliken charge as small as 0.01 $|e|$. From the fifth shell the charge oscillations take place. Stars VI and VII are twofold degenerate, and net charges for the two sets are quite different and of opposite sign: 0.114 and -0.054 (12 and 12 atoms) and 0.112 and -0.057 (4 and 12 atoms) for stars VI and VII, respectively. In Table 1 the mean value for each shell of neighbors is reported. Concerning the $\mathrm{C}_{455} \mathrm{H}_{196}$ cluster, the net charge is essentially null for the first 5 stars ( 35 atoms) of neighbors, and in 6 additional stars (overall 125 atoms) it is smaller than $0.01|e|$. From the very large $\mathrm{C}_{651} \mathrm{H}_{252}$ cluster on, the net charges are null for all the first seven shells of neighbors.

Eigenvalues (the "band structure"), on the contrary, are very sensitive to the boundary conditions, since they are affected by the long range interactions between electrons and between electrons and the lattice (i.e. the real electrostatic potential of the crystal): the lack of the infinite crystal lattice

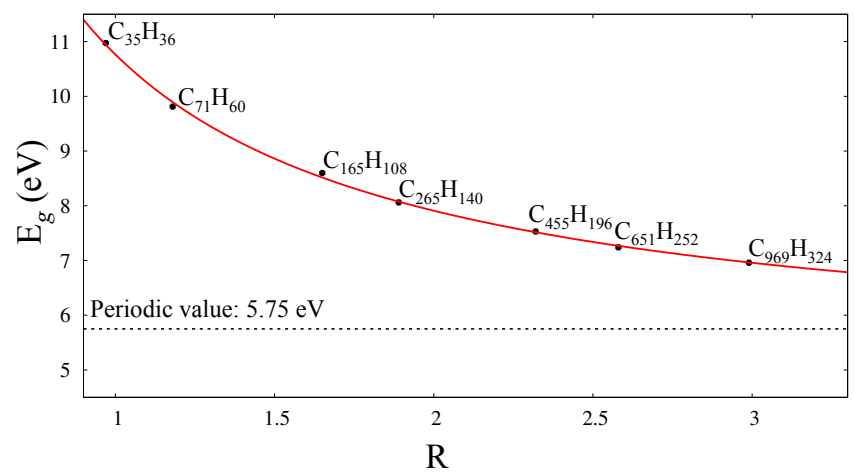

Fig. 2 Energy gap (HOMO-LUMO distance, in eV) of non defective diamond computed with molecular cluster of increasing size. Values have been fitted with a hyperbolic function of R. Periodic $\mathrm{E}_{g}$ value computed with $S_{128}$ (dashed line) is also reported for comparison. Results from spin restricted calculations. 


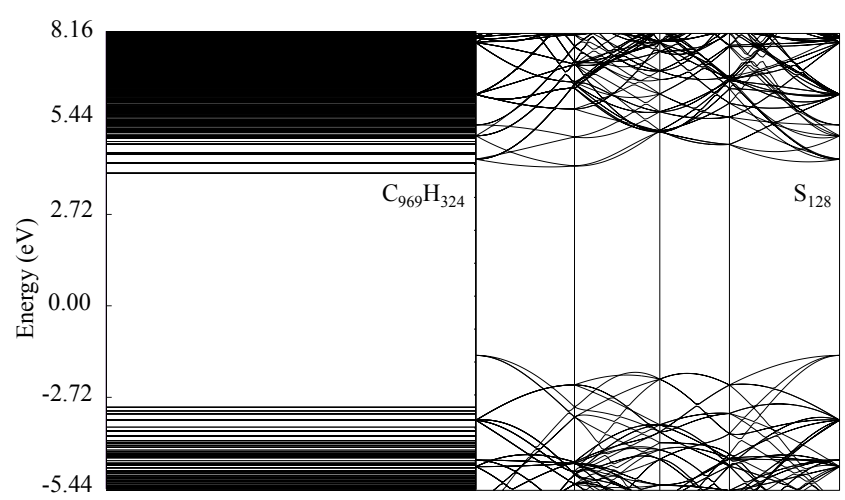

Fig. 3 Energy levels and band structure (along the $\Gamma \rightarrow X \rightarrow L \rightarrow$ $W \rightarrow \Gamma$ path) of the pristine diamond cluster $\left(\mathrm{C}_{969} \mathrm{H}_{324}\right.$, left panel) and supercell $\left(\mathrm{S}_{128}\right.$, right panel), respectively.

cannot be easily recovered by increasing the cluster size [37, 38]. A clear example of this behavior is the HOMO-LUMO gap $\left(\mathrm{E}_{g}\right)$ that is reported in Figure 2 as a function of the $\mathrm{C} / \mathrm{H}$ ratio, $\mathrm{R}$, (the corresponding periodic value is also reported for comparison).

The Figure shows a very slow convergence with $\mathrm{R}$ towards a limiting value. The $\mathrm{C}_{71} \mathrm{H}_{60}$ gap is about $4 \mathrm{eV}$ larger than the corresponding periodic value (dashed line in Figure 2); for the two subsequent clusters the difference reduces by about $1 \mathrm{eV}$ at each step. For two even larger clusters, $\mathrm{C}_{651} \mathrm{H}_{252}$ and $\mathrm{C}_{969} \mathrm{H}_{324}$, the gap reduces each time by $0.3 \mathrm{eV}$ to 6.96 $\mathrm{eV}$, however remaing still $1.2 \mathrm{eV}$ larger than the bulk value at $5.76 \mathrm{eV}$ (5.99 eV for the direct gap). By fitting the seven points shown in the figure with the following equation

$\mathrm{E}_{g}=\frac{a}{\mathrm{R}}+b$

The value of the $b$ coefficient is around $5 \mathrm{eV}$, with a \pm 0.07 $\mathrm{eV}$ oscillation when subsets of points are used for the fit. This means that the gap for an infinite cluster is about 0.7 $\mathrm{eV}$ smaller than the one of the bulk $(5.75 \mathrm{eV})$. The analysis of the HOMO and LUMO bands (see Figure 3) shows that actually the contribution of the border carbon atoms (the ones partially linked to hydrogen atoms) is far from negligible, with some participation of the hydrogen atoms too. In summary, the cluster band gaps remain affected by border effects whichever the size of the cluster is.

One of the most important techniques for identifying defects in diamond is vibrational spectroscopy, in particular the Raman one. Pure diamond is characterized by a single Raman peak at $1332 \mathrm{~cm}^{-1}$; the same peak, computed within the periodic approach is at $1318 \mathrm{~cm}^{-1}$, see Refs.[39-41] for a detailed discussion. However, when a cluster approach is adopted, spurious peaks due to border effects are expected. Thefore, before considering the Raman spectrum of the defective diamond (how many new peaks appear? Above or below the peak at $1318 \mathrm{~cm}^{-1}$ ?) we must check to which extent the boundary conditions affect the cluster spectra and how large must be the cluster for providing the bulk peak. Figure 4 (left panels, black curves) reports the Raman spectra of the $\mathrm{C}_{71} \mathrm{H}_{60}, \mathrm{C}_{165} \mathrm{H}_{100}$ and $\mathrm{C}_{265} \mathrm{H}_{140}$ clusters. These spectra have been obtained by computing the FULL spectrum of the cluster, optimizing the geometry of all atoms but the terminal $\mathrm{H}$ atoms. Thus, looking at the Figure, it turns out that, unlike the periodic case, there are many peaks in the $1200-1400 \mathrm{~cm}^{-1}$ region of the spectrum: a twin peak at 1286-1305 together with a sharp one at around $1365 \mathrm{~cm}^{-1}$ for $\mathrm{C}_{71} \mathrm{H}_{60}$; several peaks at 1294, 1311,1331, 1378 and a very intense one at $1344 \mathrm{~cm}^{-1}$ for $\mathrm{C}_{165} \mathrm{H}_{100}$; a sharp peak at $1294 \mathrm{~cm}^{-1}$, that dominates the spectrum, but accompanied by several less intense peaks at higher energies (at 1313, $1354,1381 \mathrm{~cm}^{-1}$ ) for $\mathrm{C}_{265} \mathrm{H}_{140}$.

Several main points can be raised when looking at the figure: i) which peak is the "true" one of pristine diamond? ii) Is it possible to discriminate spurious peaks due to border effects from the one of the diamond bulk? iii) How large should the cluster be in order to provide a reliable spectrum? In order to try to answer to these questions, we manipulated in two ways the spectrum:

- through the ${ }^{12} \mathrm{C} \rightarrow{ }^{100} \mathrm{C}$ isotopic substitution (see the option ISOTOPES in Ref.[44]): an unphysical mass of 100 a.m.u. has been attributed to all the carbon atoms that are connected to hydrogens, in order to eliminate their contribution from the modes. The resulting spectra are shown in Figure 4, (middle panels, red lines)

- limiting the vibrational analysis to a fragment of the cluster (see the "Computational details" section). The FRAGMENT procedure has been applied only to the $\mathrm{C}_{165} \mathrm{H}_{108}$ and $\mathrm{C}_{265} \mathrm{H}_{140}$ clusters $\left(\mathrm{C}_{71} \mathrm{H}_{60}\right.$ being too small): in these cases the Raman spectra has been computed only for the central core of respectively 35 and $71 \mathrm{C}$ atoms that has been fully relaxed. Computed spectra are shown in Figure 4, (right panels, blue lines).

When applying the isotopic substitution, the resulting spectra are largely simplified. Only one peak, at $1203 \mathrm{~cm}^{-1}$, remains for the $\mathrm{C}_{71} \mathrm{H}_{60}$ cluster; two peaks at 1290 (intense) and 1262 (weak) $\mathrm{cm}^{-1}$ for $\mathrm{C}_{165} \mathrm{H}_{100}$; two peaks at 1292 (intense) and 1261 (slightly less intense) $\mathrm{cm}^{-1}$ for $\mathrm{C}_{265} \mathrm{H}_{140}$. The peak at $1203 \mathrm{~cm}^{-1}$ of $\mathrm{C}_{71} \mathrm{H}_{60}$ shows a large isotopic shift, thus indicating that the normal coordinate is largely dominated by border effects: this frequency cannot be recognized as a "bulk" mode of diamond since it is mainly the vibration of a small central nucleus ( $5 \mathrm{C}$ atoms) againts the wall of the heavy $\mathrm{C}$. In the $\mathrm{C}_{165} \mathrm{H}_{100}$ and $\mathrm{C}_{265} \mathrm{H}_{140}$ clusters the peaks at higher energy (1290 and $\left.1292 \mathrm{~cm}^{-1}\right)$ are actually the "bulk" like collective motions of C as computed with periodic calculations (note that the peak at $1292 \mathrm{~cm}^{-1}$ of $\mathrm{C}_{265} \mathrm{H}_{140}$ is not shifted by applying the isotopic substitution; the other two signals (1262 and $1261 \mathrm{~cm}^{-1}$ ) are again 


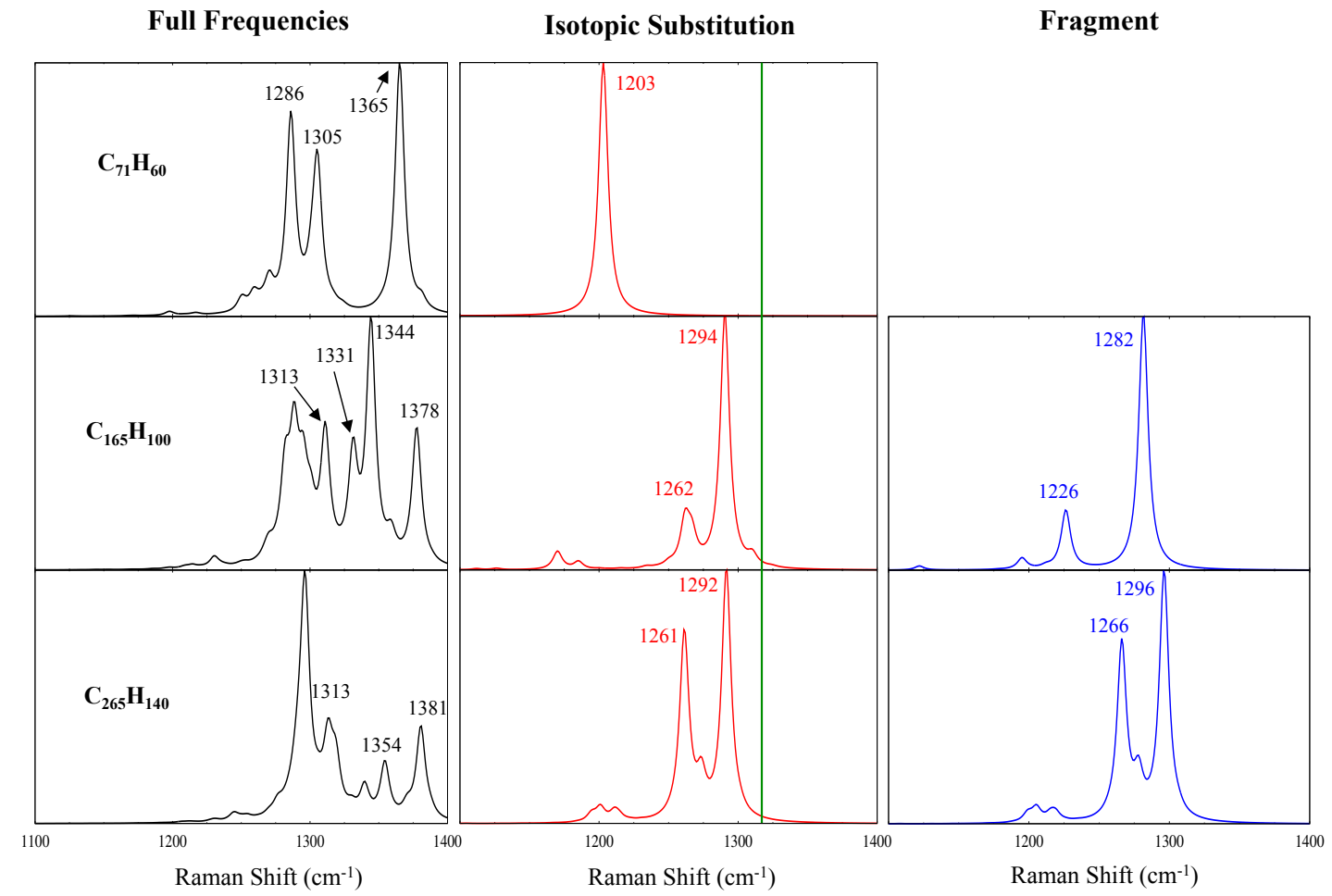

Fig. 4 Raman spectra of three different clusters obtained by fully relaxing the structure but the hydrogen atoms (left and center panels, the latter obtained through the isotopic substitution ${ }^{12} \mathrm{C} \rightarrow{ }^{100} \mathrm{C}$ ) and relaxing only a few inner shells of atoms (right panels). See text for details. Results from spin restricted calculations.

Table 2 Cluster and periodic relaxation energies $E_{r}$ (mHa) and outwards displacement ( $d$, in $\AA$ ) obtained by relaxing, when available, up to five stars of neighbors around the vacancy defect. Full values for the periodic case are obtained by optimizing both internal coordinates and lattice parameter. All results are from spin unrestricted calculations and refer to the $S_{z}=0(M=1)$ magnetic state.

\begin{tabular}{l|lcccc|c}
\hline \hline & Neighbors & $\mathrm{C}_{35} \mathrm{H}_{36}$ & $\mathrm{C}_{71} \mathrm{H}_{60}$ & $\mathrm{C}_{165} \mathrm{H}_{100}$ & $\mathrm{C}_{455} \mathrm{H}_{196}$ & $\mathrm{~S}_{128}$ \\
\hline \multirow{6}{*}{$E_{r}$} & 4 & 28.61 & 26.62 & 27.12 & 26.74 & 25.79 \\
& $4+12$ & 29.00 & 27.76 & 28.23 & 28.24 & 27.25 \\
& $4+12+12$ & - & 28.07 & 28.45 & 28.38 & 27.67 \\
& $4+12+12+6$ & - & 28.10 & 28.62 & 28.41 & 27.71 \\
& $4+12+12+6+12$ & - & 28.94 & 29.56 & 29.37 & 28.52 \\
& Full & 29.30 & 29.00 & 29.95 & 29.90 & 28.98 \\
\hline \multirow{6}{*}{$d$} & 4 & 0.138 & 0.118 & 0.118 & 0.118 & 0.116 \\
& $4+12$ & 0.131 & 0.117 & 0.112 & 0.099 & 0.109 \\
& $4+12+12$ & - & 0.118 & 0.113 & 0.111 & 0.109 \\
& $4+12+12+6$ & - & - & 0.113 & 0.111 & 0.109 \\
& $4+12+12+6+12$ & - & - & 0.125 & 0.117 & 0.114 \\
\hline \hline
\end{tabular}

"border" modes (all the spurius peaks seem to collapse in this mode): they can be mainly described as the vibrations at the interface between the core of the cluster and the surrounding heavy $\mathrm{C}$. This analysis is corroborated by results obtnained through the FRAGMENT option. For $\mathrm{C}_{165} \mathrm{H}_{100}$ and $\mathrm{C}_{265} \mathrm{H}_{140}$ the Raman peaks are grouped again into two main peaks at 1226 and $1282 \mathrm{~cm}^{-1}$ for the former, and at1266 and $1296 \mathrm{~cm}^{-1}$ for the latter cluster. Note that the Raman spectra computed with the FRAGMENT procedure are fully comparable with those derived through the isotopic substitution. With an even larger cluster it is expected that the peak at $1266 \mathrm{~cm}^{-1}$ of the FRAGMENT spectra of the $\mathrm{C}_{265} \mathrm{H}_{140}$ cluster will further move to about $1290 \mathrm{~cm}^{-1}$, showing then a spectrum similar to the bulk one.

Our analysis has highlighted some critical issues in the Raman spectra computed with clusters. First of all, the periodic spectrum of the pure diamond is never reproduced, at least with the clusters studied in this work (only starting from the large $\mathrm{C}_{265} \mathrm{H}_{140}$ the computed spectrum is vaguely comparable to the periodic one). Moreover, the reliability of the spectra requires well aimed manipulations in order to discriminate "true" spectroscopic peaks from "border" features that 
Table 3 Distances between neighbors $(d$, in $\AA$ ) and spin density $(\mu$, in $|e|)$ in the region near to the the vacancy and the intestitial defects. All results refer to $S_{z}=0$ spin state $(M=1)$. The interatomic C-C distance in the perfect system is $1.561 \AA$. See Figure 5 for labelling of atoms. All results are from spin unrestricted calculations and refer to the $S_{z}=0$ spin state $(M=1)$.

\begin{tabular}{c|c|cccc|c}
\hline \hline Vacancy & & $\mathrm{C}_{35-1} \mathrm{H}_{36}$ & $\mathrm{C}_{71-1} \mathrm{H}_{60}$ & $\mathrm{C}_{165-1} \mathrm{H}_{100}$ & $\mathrm{C}_{455-1} \mathrm{H}_{196}$ & $\mathrm{~S}_{128-1}$ \\
\hline \multirow{4}{*}{$d$} & $\mathrm{~V}-\mathrm{C}_{1}$ & 1.693 & 1.684 & 1.686 & 1.685 & 1.656 \\
& $\mathrm{C}_{1}-\mathrm{C}_{2}$ & 1.511 & 1.512 & 1.514 & 1.517 & 1.524 \\
& $\mathrm{C}_{2}-\mathrm{C}_{3}$ & 1.559 & 1.558 & 1.563 & 1.563 & 1.564 \\
\hline \multirow{4}{*}{$\mu$} & $\mathrm{C}_{1}$ & \pm 0.808 & \pm 0.751 & \pm 0.732 & \pm 0.723 & \pm 0.710 \\
& $\mathrm{C}_{2}$ & $\mp 0.055$ & $\mp 0.048$ & $\mp 0.049$ & $\mp 0.049$ & $\mp 0.047$ \\
& $\mathrm{C}_{3}$ & \pm 0.016 & \pm 0.014 & \pm 0.015 & \pm 0.015 & \pm 0.015 \\
\hline \hline Interstitial & & $\mathrm{C}_{35+1} \mathrm{H}_{36}$ & $\mathrm{C}_{71+1} \mathrm{H}_{60}$ & $\mathrm{C}_{165+1} \mathrm{H}_{100}$ & $\mathrm{C}_{455+1} \mathrm{H}_{196}$ & $\mathrm{~S}_{128+1}$ \\
\hline \multirow{4}{*}{$d$} & $\mathrm{C}_{-} \mathrm{C}$ & 1.291 & 1.300 & 1.303 & 1.304 & 1.303 \\
& $\mathrm{C}_{1} \mathrm{C}_{1}$ & 1.424 & 1.424 & 1.424 & 1.425 & 1.421 \\
& $\mathrm{C}_{1}-\mathrm{C}_{2}$ & 1.517 & 1.519 & 1.519 & 1.519 & 1.517 \\
& $\mathrm{C}_{2}-\mathrm{C}_{3}$ & 1.555 & 1.576 & 1.581 & 1.581 & 1.580 \\
\hline \multirow{4}{*}{$\mu$} & $\mathrm{C}$ & \pm 1.043 & \pm 1.004 & \pm 1.004 & \pm 1.003 & \pm 0.840 \\
& $\mathrm{C}_{1}$ & $\mp 0.122$ & $\mp 0.123$ & $\mp 0.123$ & $\mp 0.123$ & $\mp 0.032$ \\
& $\mathrm{C}_{2}$ & \pm 0.028 & \pm 0.032 & \pm 0.033 & \pm 0.033 & \pm 0.018 \\
\hline \hline
\end{tabular}

unfortunately lie in the same spectral range. Finally, even when a "bulk" like mode can be identified in the spectrum, the peak differs by at least $20 \mathrm{~cm}^{-1}$ with respect to the periodic value (1292 vs $1318 \mathrm{~cm}^{-1}$ ).

\subsection{Defective Diamond}

Once the molecular cluster is extracted from the optimized diamond structure, a vacancy or an insterstitial defect is created through the substitution of the central carbon with a ghost atom or by addition of an interstitial carbon near the central one, see Figure 5. The formation of a vacancy in a diamond generates four dangling bonds at the nearest neighbors. The other electrons of these atoms are involved in very strong covalent bonds, so that the electrons occupying the dangling bonds can just couple to each other, generating (in a monodeterminantal view) states with $\mathrm{S}_{z}=2$ (four $\alpha$ spin electrons), $\mathrm{S}_{z}=1$ (three $\alpha$ and one $\beta$ ) and $\mathrm{S}_{z}=0$ (two $\alpha$ and two $\beta$ ).

Concerning the interstitial defect, the $\langle 100\rangle$ split self-interstitial model (the most stable one) is characterized by a short bond distance between the two central carbon atoms

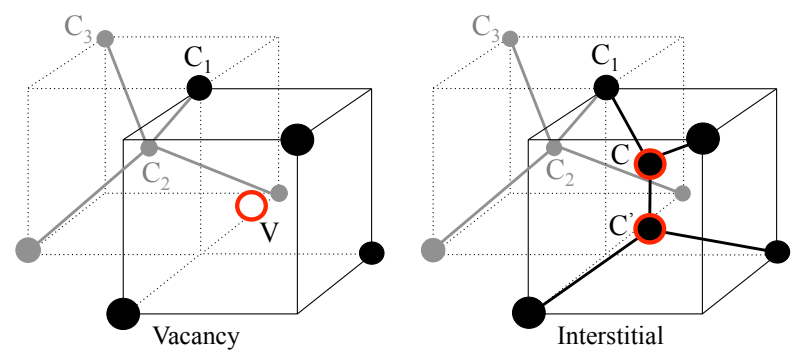

Fig. 5 Schematic representation of the vacancy and of the interstitial defective sites.
(1.30 ̊), aligned along the $\langle 100\rangle$ crystallographic direction, Figure 5. The constraint from the lattice does not permit the formation of a double bond and unpaired electrons lie well localized at the defect site, leading to singlet $S_{z}=0$ or triplet $S_{z}=1$ magnetic states.

Thanks to the very local nature of these defects, cluster and periodic approaches are expected to converge to the same values for properties such as spin density, relaxation and formation energies, energy differences between various spin states and HOMO-LUMO (band) gaps. Thus, in the following, these properties computed with clusters of different size are analized and compared to the $S_{128}$ results.[39,40,62]

In Table 2 the relaxation energy, $\mathrm{E}_{r}$, of the neutral vacancy, obtained by optimizing the position of an increasing number of shells of neighbors, is reported. The Table shows that quite similar values have been computed for the various clusters (the exception being the smallest one, $\mathrm{C}_{35} \mathrm{H}_{36}$ ) and for the periodic calculation, even if $\mathrm{E}_{r}$ computed with clusters are in general larger, by about $1 \mathrm{mHa}(30 \mathrm{meV})$, than the periodic counterparts. The largest energy gain is obtained by the relaxation of the first neighbors, while all subsequent relaxations (up to the fifth shell) increase the relaxation energy by less than $10 \%$ (about $0.05 \mathrm{eV}$ ). When a full relaxation of the carbon atoms is performed, only $0.46 \mathrm{mHa}(15 \mathrm{meV})$ are gained (last row of Table 2). The back relaxation of the nearest shells of neighbors, also shown in Table 2, confirms the rapid convergence with the cluster size, and the quite good agreement with supercell results. Such a local behavior is even clearer when the interatomic bond lenghts are considered, (reported in Table 3 for both defects); the perturbation induced by the defect to the interatomic distances is significant only up to 3-4 stars of neighbors, where the value of pristine diamond (1.561 $\AA$ ) is basically reached. It is not surprising thus that all clusters provide distances in excellent agreement with the periodic calculation. 
Spin density, identifying the magnetic states of the defects, is also a very local property, and all clusters, irrespective of their size, provide results in line with those from periodic calculations, see Table 3 . Concerning the neutral vacancy, spin density is localized on the first four $\mathrm{C}$ atoms surrounding the defect, with a spin charge that varies from $0.808|e|$ for $\mathrm{C}_{35-1} \mathrm{H}_{36}$ to $0.723|e|$ for $\mathrm{C}_{455-1} \mathrm{H}_{196}$ clusters, and practically vanishes from third neighbors onwards. As regards the $\langle 100\rangle$ split self-interstitial defect, the spin density is found to be localized mainly on the two central carbon atoms, C-C' (Figure 5), with values that range from 1.043 to $1.001|e|$ when the smallest $\mathrm{C}_{35+1} \mathrm{H}_{36}$ and largest $\mathrm{C}_{455+1} \mathrm{H}_{196}$ studied clusters are considered: the spin density propagates with a sign alternation in a rather damped way to first-neighbors, and again almost vanishes from third neighbors onwards. The good agreement between cluster and periodic approaches is also well documented in Figure 6 , where the spin density maps of the $S_{z}=0$ spin state of the vacancy, obtained with the $\mathrm{C}_{165-1} \mathrm{H}_{100}$ cluster and the $\mathrm{S}_{128-1}$ supercell, are compared.

In Table 4 the energetics of the defects is reported: $\mathrm{E}_{f}$ is the formation energy; $\mathrm{E}_{0-1}$, the energy difference between the open shell spin states $S_{z}=0$ and $S_{z}=1, E_{0-2}$ the energy difference between the open shell spin states $S_{z}=0$ and $S_{z}=2$, $\mathrm{E}_{0-C S}$ the energy difference between the open shell spin state $S_{z}=0$ and the closed shell solution. All these energy dif-
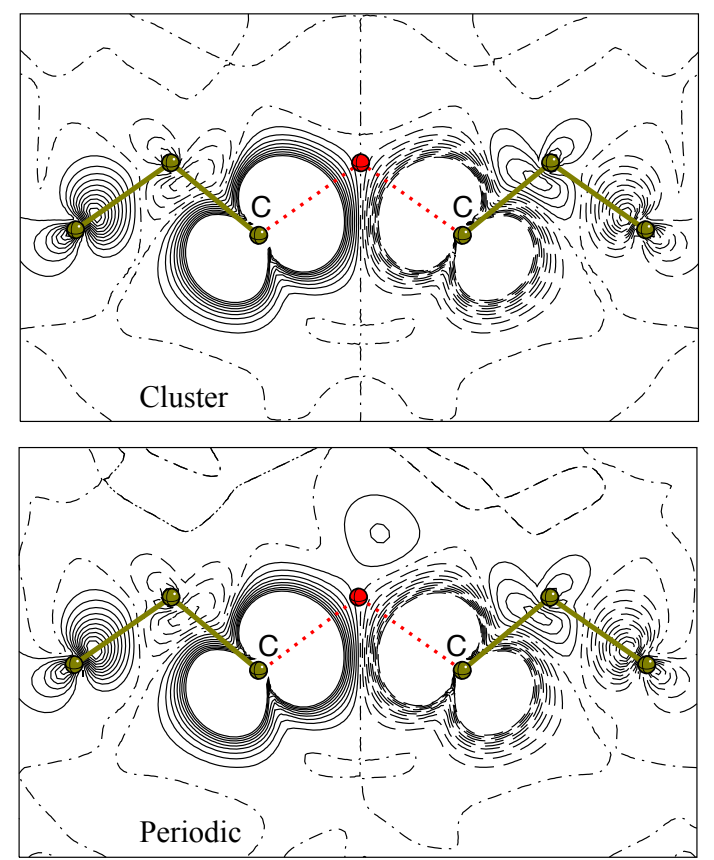

Fig. 6 Cluster and periodic spin density maps for the $S_{z}=0$ state. Red atom and dashed lines represent the vacancy and the dangling bonds, respectively. Isodensity lines differ by $0.01|\mathrm{e}| /\left(a_{0}\right)^{3}$, while spin densities are truncated at $\pm 0.1|\mathrm{e}| /\left(a_{0}\right)^{3}$. Continuous, dashed and dot-dashed lines denote respectively positive, negative and zero values. ferences converge very fast with cluster size to values quite close to the supercell ones. Moreover, all clusters calculations predict correctly the $S_{z}=0$ state as the most stable spin configuration, and that the small energy differences $\mathrm{E}_{0-1}$ for the defects are computed with a discrepancy on only few tenths of $\mathrm{mHa}$ with respect to the periodic calculations. In addition, starting from the $\mathrm{C}_{165 \pm 71} \mathrm{H}_{120}$ cluster, all the other energy values reported in Table 4 differ from the supercell counterparts at most for $8 \mathrm{mHa}(0.2 \mathrm{eV})$.

Another interesting comparison that can be done regards cluster and periodic energy spectra. In Figure 7 cluster and $\mathrm{S}_{128}$ "band" structures of the neutral vacancy and the interstitial defects, both in their $S_{z}=0$ most stable spin state, are reported. The main effect of the defect on the band structure is just to split the main gap into three (interstitial), four (vacancy) subintervals (marked in the corresponding panel of Figure 7), while the main energy gap, that is the gap between the "valence" and the "conduction" band $\left(\mathrm{E}_{g}^{D}=5.85\right.$ $\mathrm{eV}$ ), basically remains unaltered with respect to pure diamond $\left(\mathrm{E}_{g}=5.75 \mathrm{eV}\right)$. Clusters provide a qualitavely nice description of the overall shape of the band structure, once providing that clusters of reasonable size (starting from $\mathrm{C}_{165 \pm 1^{-}}$ $\mathrm{H}_{100}$ ) are employed. Nonetheless, convergence of cluster energy values towards supercell results is very slow (compare Figure 7 and values in Table 5). For instance for $\mathrm{C}_{165 \pm 1^{-}}$ $\mathrm{H}_{100}$ and $\mathrm{C}_{265 \pm 1} \mathrm{H}_{140}$, that have been shown to provide a very good description of geometry and energetics of the perturbed systems, $\mathrm{E}_{h C}$ (that is the energy difference between the highest defective level and the conduction band) are respectively 3.33 and $3.07 \mathrm{eV}$ (the supercell value is $0.74 \mathrm{eV}$ ) for the vacancy, 2.32 and $2.13 \mathrm{eV}$, respectively, for the interstitial defect (the supercell value is $1.45 \mathrm{eV}$ ). This implies that any discussion concerning gap size and position of defect states in the gap must take into account this overestimation.

Finally, we turn then our analysis to the Raman spectrum of the defective systems. At variance with what has been shown in the case of the neutral vacancy in diamond (where no significant Raman peaks related to the defect are found far from that spectroscopic region), the $\langle 100\rangle$ split self-interstitial defect produces a couple of peaks above the pristine diamond band: the first peak is at about $1500 \mathrm{~cm}^{-1}$, the second one around $1900 \mathrm{~cm}^{-1}$ (a detailed analysys of vibrational properties of defective diamond can be found in Refs.[39,41]). The highest-frequency vibrational mode is the bond stretching of the two central carbon atoms of the defect. The other normal mode corresponds to the asymmetric stretching involving the two central atoms and their first-neighbors (see Figure 5).

Raman spectra computed with $\mathrm{C}_{265+1} \mathrm{H}_{140}$ and $\mathrm{S}_{128}$ are compared in Figure 8. The $\mathrm{S}_{128}$ spectrum exhibits the two signals of the defect at $1529 \mathrm{~cm}^{-1}$ and $1919 \mathrm{~cm}^{-1}$ and the pristine diamond peak splitted into three main components centered 

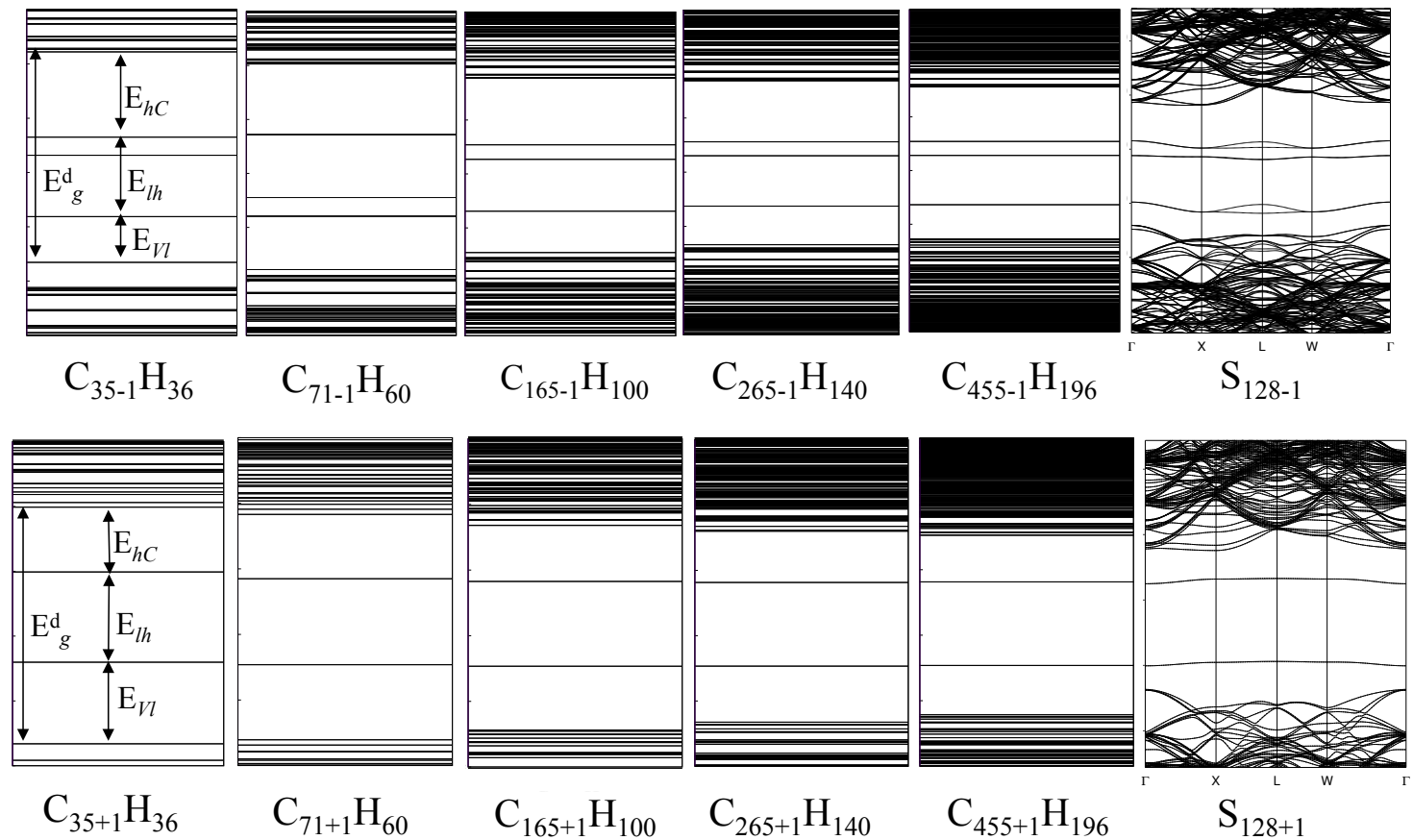

Fig. 7 Comparison between energy levels of different defective molecular clusters of increasing sizes and periodic supercell (neutral vacancy in the upper panels; $\langle 100\rangle$ split self-interstitial in the lower panels). $\mathrm{E}_{V l}$ is the difference between the "valence" band $(V)$ and the lowest defective level $(l), \mathrm{E}_{l h}$ the difference between the the lowest and the highest $(h)$ defective level, $\mathrm{E}_{h C}$ between the highest defective level and the conduction band $(C)$. Finally, $\mathrm{E}_{g}^{D}$ is the gap between the "valence" and the "conduction" band. All results are from spin unrestricted calculations for the most stable $\mathrm{S}_{z}=0(\mathrm{M}=1)$ magnetic state.

at $1276,1307,1337 \mathrm{~cm}^{-1}$. This split can be brought back to the $T_{d} \rightarrow C_{2 v}$ local symmetry reduction. As regards the cluster spectrum, it shows the two modes of the defect well localized at $1516 \mathrm{~cm}^{-1}$ and $1917 \mathrm{~cm}^{-1}$ and a group of bands in the $1280-1400 \mathrm{~cm}^{-1}$ spectral range, where as demonstrated in the previous (see Figure 4) section "bulk" and "border" modes are overlapped. In this case the ${ }^{12} \mathrm{C} \rightarrow{ }^{100} \mathrm{C}$ isotopic substitution helps only marginally. The manipulation leaves unaltered the peaks at $1516 \mathrm{~cm}^{-1}$ and $1917 \mathrm{~cm}^{-1}$, confirming the localized nature of these modes assigned to the defective site, while the $1280-1400 \mathrm{~cm}^{-1}$ bands are shifted to lower wavenumbers and their structure is simplified (now in this region five groups of peaks centered at 1339, 1310, 1291,1261 and $1215 \mathrm{~cm}^{-1}$ can be identified). Indeed the procedure is not able to completely filter spurious signals and does not permit to safely assign peaks to "bulk" diamond modes. Overall the cluster is able to provide a nice description of the spectroscopic features of the defect mainly because the corresponding peaks are far from the 1100-1400 $\mathrm{cm}^{-1}$ bulk region.

\section{Conclusions}

In this work performances of a cluster approach have been compared with those of a periodic one by analysing several properties of pure and defective diamond (defect formation energies, atomic relaxations, band structures,relative energies of different spin states, Raman spectra). Regarding the defective systems, neutral vacancy and $\langle 100\rangle$ split self-interstitial defects have been considered. Clusters of increasing size have been selected and the computed properties have been compared with those from the $\mathrm{S}_{128}$ supercell.

All calculations have been performed using the same computer code (CRYSTAL), same basis set and DFT functional. In this way, differences between the results of the two methods can be attributed completely to the assumption of cluster or periodic boundary conditions, rather than to differences in the Hamiltonian, basis set or computational scheme. Since the effect of the finite size of the cluster is not the same for all properties, we have verified how deeply the border perturbation propagates inside the cluster and how it affects the physical picture provided by the molecular systems.

The creation of a defect produces a perturbation in the geometric, electron and magnetic properties of pristine diamond that remains localized near the defective site, vanishing within 3-4 shells of neighbors. As a consequence, all the related properties (geometry, energetics, magnetic structure) converge rapidly as the size of the cluster increases, and already a small cluster as $\mathrm{C}_{71 \pm 1} \mathrm{H}_{60}$ provides a good description of the defect. With $\mathrm{C}_{265 \pm 1} \mathrm{H}_{140}$ properties are almost converged and in excellent agreement with those predicted by the periodic calculation. 
Table 4 Energy differences (in $\mathrm{mHa}$ ) between different possible spin states of both defective structures, obtained with cluster and supercell approaches. $E_{0-1}$ and $E_{0-2}$ are the energy differences between the open-shell $S_{z}=0(M=1)$ and $S_{z}=1 \quad(M=3)$ and $S_{z}=0(M=1)$ and $S_{z}=2(M=5)$ spin states, respectively. $\mathrm{E}_{0-C S}$, only defined for the interstitial defect, is the energy difference between the open-shell singlet spin state and the corresponding closed shell solution. Finally, $\mathrm{E}_{f}$ is the formation energy of the defect (in eV) computed as: $\mathrm{E}_{f}=\mathrm{E}_{P}-\mathrm{E}_{D}+\mathrm{E}_{A}\left(\right.$ vacancy) and $\mathrm{E}_{f}=\mathrm{E}_{P^{-}}$ $\mathrm{E}_{D}-\mathrm{E}_{A}$ (interstitial) where $\mathrm{E}_{P}$ and $\mathrm{E}_{D}$ are the total energies of prestine and defective diamond, respectively, and $\mathrm{E}_{A}$ is the atomic energy (see also Refs.[40,41]).

\begin{tabular}{cccccc|c}
\hline \hline Vacancy & $\mathrm{C}_{35-1} \mathrm{H}_{36}$ & $\mathrm{C}_{71-1} \mathrm{H}_{60}$ & $\mathrm{C}_{165-1} \mathrm{H}_{100}$ & $\mathrm{C}_{265-1} \mathrm{H}_{140}$ & $\mathrm{C}_{455-1} \mathrm{H}_{196}$ & $\mathrm{~S}_{128-1}$ \\
\hline $\mathrm{E}_{0-1}$ & 6.1 & 5.6 & 5.3 & 5.2 & 5.1 & 5.0 \\
$\mathrm{E}_{0-2}$ & 56.95 & 57.26 & 56.57 & 56.51 & 56.50 & 51.8 \\
$\mathrm{E}_{f}$ & 7.24 & 7.01 & 6.91 & 6.88 & 6.86 & 6.74 \\
\hline \hline Interstitial & $\mathrm{C}_{35+1} \mathrm{H}_{36}$ & $\mathrm{C}_{71+1} \mathrm{H}_{60}$ & $\mathrm{C}_{165+1} \mathrm{H}_{100}$ & $\mathrm{C}_{265+1} \mathrm{H}_{140}$ & $\mathrm{C}_{455+1} \mathrm{H}_{196}$ & $\mathrm{~S}_{128+1}$ \\
\hline $\mathrm{E}_{0-1}$ & 0.95 & 0.83 & 0.85 & 0.85 & 0.85 & 1.1 \\
$\mathrm{E}_{0-C S}$ & 79.20 & 66.40 & 65.72 & 65.34 & 65.08 & 57.6 \\
$\mathrm{E}_{f}$ & 12.39 & 11.78 & 11.29 & 11.08 & 10.79 & 10.45 \\
\hline \hline
\end{tabular}

Table 5 Energy differences (in eV) between intra-gap levels for the vacancy and the interstitial molecular clusters. $\mathrm{E}_{V l}$ is the difference between the "valence" band $(V)$ and the lowest defective level $(l), \mathrm{E}_{l h}$ the difference between the the lowest and the highest $(h)$ defective level, $\mathrm{E}_{h C}$ between the highest defective level and the conduction band $(C)$. Finally, $\mathrm{E}_{g}^{D}$ is the gap between the "valence" and the "conduction" band. All results are from spin unrestricted calculations and refer to the $S_{z}=0$ spin state $(M=1)$.

\begin{tabular}{c|c|ccccc|c}
\hline \hline Vacancy & & $\mathrm{C}_{35-1} \mathrm{H}_{36}$ & $\mathrm{C}_{71-1} \mathrm{H}_{60}$ & $\mathrm{C}_{165-1} \mathrm{H}_{100}$ & $\mathrm{C}_{265-1} \mathrm{H}_{140}$ & $\mathrm{C}_{455-1} \mathrm{H}_{196}$ & $\mathrm{~S}_{128-1}$ \\
\hline & $\mathrm{E}_{V l}$ & 2.28 & 2.66 & 2.07 & 1.94 & 1.72 & 1.52 \\
& $\mathrm{E}_{l h}$ & 3.09 & 2.73 & 2.60 & 2.53 & 2.51 & 2.14 \\
& $\mathrm{E}_{h C}$ & 4.25 & 3.13 & 3.33 & 3.07 & 2.77 & 0.74 \\
& $\mathrm{E}_{g}^{D}$ & 10.50 & 10.27 & 8.72 & 8.23 & 7.70 & 5.97 \\
\hline \hline Interstitial & & $\mathrm{C}_{35+1} \mathrm{H}_{36}$ & $\mathrm{C}_{71+1} \mathrm{H}_{60}$ & $\mathrm{C}_{165+1} \mathrm{H}_{100}$ & $\mathrm{C}_{265+1} \mathrm{H}_{140}$ & $\mathrm{C}_{455+1} \mathrm{H}_{196}$ & $\mathrm{~S}_{128+1}$ \\
\hline & $\mathrm{E}_{V l}$ & 3.42 & 3.12 & 2.64 & 2.33 & 1.94 & 0.98 \\
& $\mathrm{E}_{l h}$ & 3.79 & 3.57 & 3.51 & 3.49 & 3.47 & 3.41 \\
& $\mathrm{E}_{h C}$ & 2.72 & 2.67 & 2.32 & 2.13 & 2.05 & 1.45 \\
& $\mathrm{E}_{g}^{D}$ & 9.93 & 9.35 & 8.48 & 7.96 & 7.47 & 5.85 \\
\hline \hline
\end{tabular}

The situation is different for the "band" structures: energy levels are very sensitive to the boundary conditions, i.e. to the finite size at the cluster. Indeed for pure diamond, $\mathrm{E}_{g}$ converges very slowly with cluster size (even for the huge $\mathrm{C}_{969} \mathrm{H}_{324}$ cluster, $\mathrm{E}_{g}$ is still $1.2 \mathrm{eV}$ larger than the bulk value), and, even worse, it converges to a value that is $0.7 \mathrm{eV}$ lower than the bulk value (5.0 vs $5.75 \mathrm{eV}$ ). Similar errors are observed for the intra-gap levels due to the presence of defective sites.

When collective properties as the vibrational normal modes are considered, performances of clusters are less satisfactory. On one side there is the problem of spurious features. The perturbation due to the truncation of the infinite lattice affects the Raman spectrum, and causes the presence of very intense "border" peaks that overlap the "true" diamond signals in the same spectral range, between $1100-1400 \mathrm{~cm}^{-1}$. Manipulations of the spectra, as through isotopical substitutions, can help to filter spurious peaks, but the procedure is not always completely successfull; defect features can be easlily identified only when located far away from the 1100$1400 \mathrm{~cm}^{-1}$ critical region.

On the other side there is the problem of how large must be a cluster in order to provide reliable spectra. We found that the medium size $\mathrm{C}_{71} \mathrm{H}_{60}$ is completely unable to pro- vide the collective bulk normal mode and only starting from the large $\mathrm{C}_{265} \mathrm{H}_{140}$ cluster, the computed spectrum appears vaguely comparable to that of periodic calculations. However, such a huge cluster is computationally very expensive, and does not seem to be competitive with respect to a supercell calculation.

\section{References}

1. R. W. Grimes, C. R. A Catlow, and A. L. Shluger. Quantum mechanical cluster calculations in solid state studies. World Scientific, 1992.

2. P. S. Bagus, G. Pacchioni, and F. Parmigiani. Final state effects for the core-level xps spectra of nio. Chem. Phys. Lett., 207(4):569574, 1993.

3. Z. Barandiarán and L. Seijo. The Ab-Initio Model Potential Representation of the Crystalline Environment. Theoretical Study of the Local Distortion on NaCl: Cu+. J. Chem. Phys., 89(9):5739-5746, 1988.

4. L. H. Li and J. E. Lowther. Lattice Relaxation at Vacancy Aggregates in Diamond. Phys. Rev. B, 53:11277-11280, 1996.

5. A. Mainwood. Relaxation About the Vacancy in Diamond. $J$. Phys. C: St. Phys, 11(13):2703, 1978.

6. D. Hyde-Volpe, B. Slepetz, and M. Kertesz. The $[\mathrm{V}-\mathrm{C}=\mathrm{C}-\mathrm{V}]$ divacancy and the interstitial defect in diamond: Vibrational properties. J. Phys. Chem. C, 114(21):9563-9567, 2010. 


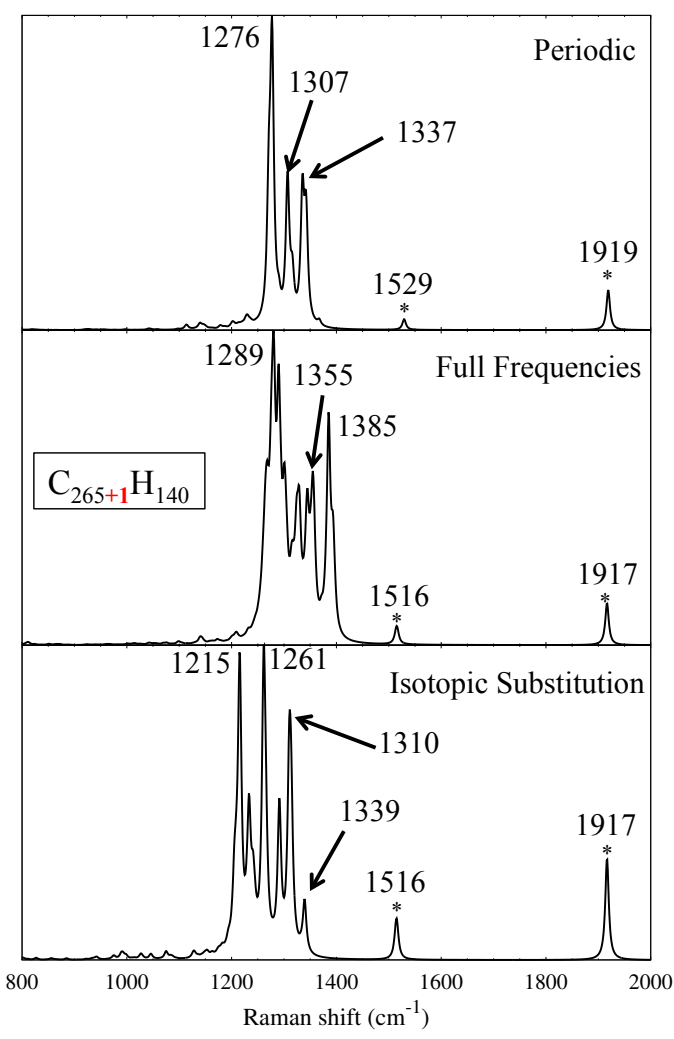

Fig. 8 Raman spectrum of the $\mathrm{C}_{265+1} \mathrm{H}_{100}$ before (upper) and after isotopic substitution ${ }^{12} \mathrm{C} \rightarrow{ }^{100} \mathrm{C}$ (lower) in which a $\langle 100\rangle$ split self-interstitial point defect has been created. The intensities of the two peaks marked with * have been multiplied by a factor 15 . All results are from spin unrestricted calculations and refer to the $S_{z}=0$ spin state $(M=1)$.

7. S. J. Breuer and P.R. Briddon. Ab Initio Investigation of the Native Defects in Diamond and Self-Diffusion. Phys. Rev. B, 51(11):6984-6994, 1995.

8. A. Gali and J. R. Maze. Ab Initio Study of the Split SiliconVacancy Defect in Diamond: Electronic Structure and Related Properties. Phys. Rev. B, 88(23):235205, 2013.

9. M. Krosnicki, A. Kedziorski, L. Seijo, and Z. Barandiarán. Ab Initio Theoretical Study on the $4 \mathrm{f} 2$ and $4 \mathrm{f} 5 \mathrm{~d}$ Electronic Manifolds of Cubic Defects in CaF2: Pr $^{3+}$. J. Phys. Chem. A, 118(2):358368, 2013.

10. G. Pacchioni. Ab initio theory of point defects in oxide materials: structure, properties, chemical reactivity. Surf. Sci., 540:63-75, 2003.

11. K. J. Boerve and L. G. M. Pettersson. Hydrogen Abstraction from Methane on a Magnesia (001) surface. J. Phys. Chem., 95(19):7401-7405, 1991.

12. Neyman K. M. and Rösch N. Bonding and vibration of co molecules adsorbed on low-coordinated surface sites of mgo: a lcgto-ldf cluster investigation. Surf. Sci., 297(2):223-234, 1993.

13. J. Sauer. Quantum mechanical studies of zeolites. In Modelling of Structure and Reactivity in Zeolites, pages 183-216. Academic Press, 1992.

14. E. H. Teunissen, F. B. Van Duijneveldt, and R. A. Van Santen. Interaction of Ammonia with a Zeolitic Proton: Ab Initio QuantumChemical Cluster Calculations. J. Phys. Chem., 96(1):366-371, 1992.

15. A. M. Ferrari, P. Ugliengo, and E. Garrone. Geminal Silica Hydroxyls as Adsorbing Sites: an Ab-Initio study. J. Phys. Chem., 97:2671-2676, 1993.
16. A. M. Ferrari, S. Huber, H. Knözinger, K. M. Neyman, and N. Rösch. Ftir spectroscopic and density functional model cluster studies of methane adsorption on mgo. J. Phys. Chem. B, 102(23):4548-4555, 1998.

17. I. Panas. Properties of Molecular Crystals by Means of Theory. Acta Crystallogr. Sec. A, 49(6):881-889, 1993.

18. J. G. Ángyán and B. Silvi. Electrostatic Interactions in ThreeDimensional Solids. Self-Consistent Madelung Potential (SCMP) Approach. J. Chem. Phys., 86(12):6957-6966, 1987.

19. L. Ojamäe and K. Hermansson. Water Molecules in Different Crystal Surroundings: Vibrational O-H Frequencies from Ab Initio Calculations. J. Chem. Phys., 96(12):9035-9045, 1992.

20. S. Y. Shashkin and W. A. Goddard III. Optical Spectrum and JahnTeller Splitting of $\mathrm{Cu}^{2+}$ sites in $\mathrm{K}_{2} \mathrm{CuF}_{4}$ Based on ab initio Studies of $\left(\mathrm{CuF}_{6}\right)^{4-}$ Clusters. Phys. Rev. B, 33(2):1353, 1986.

21. G. Pacchioni, C. Sousa, F. Illas, F. Parmigiani, and P. S. Bagus. Measures of Ionicity of Alkaline-Earth Oxides from the Analysis of ab initio Cluster Wave Functions. Phys. Rev. B, 48(16):11573, 1993.

22. I. Mehdaoui and T. Kluner. Bonding of $\mathrm{CO}$ and $\mathrm{NO}$ to $\mathrm{NiO}(100)$ : a Strategy for Obtaining Accurate Adsorption Energies. J. Phys. Chem. A, 111:13233-13237, 2007.

23. F. Illas, I. de P. R. Moreira, J. M. Bofill, and M. Filatov. Spin Symmetry Requirements in Density Functional Theory: the proper way to Predict Magnetic Coupling Constants in Molecules and Solids. Theor. Chem. Acc., 116:587-597, 2006.

24. G. Pacchioni and G. Ieranø. Ab Initio Theory of Optical Transitions of Point Defects in $\mathrm{SiO}_{2}$. Phys. Rev. B, 57:817-832, 1998.

25. M. K. Nazeeruddin, F. De Angelis, S. Fantacci, A. Selloni, G. Viscardi, P. Liska, S. Ito, B. Takeru, and M. Grätzel. Combined Experimental and DFT-TDDFT Computational Study of Photoelectrochemical Cell Ruthenium Sensitizers. J. Am. Chem. Soc., 127:1683516847, 1998.

26. J. M. Azpiroz and F. De Angelis. DFT/TDDFT Study of the Adsorption of N3 and N719 Dyes on $\mathrm{ZnO}(10 \overline{10})$ Surfaces. J. Phys. Chem. A, 118:5885-5893, 1998.

27. J. Ihm, A. Zunger, and M. L. Cohen. Momentum-Space Formalism for the Total Energy of Solids. J. Phys. C, 12(21):4409, 1979.

28. C. S. Nichols, C. G. Van de Walle, and S. T. Pantelides. Mechanisms of dopant impurity diffusion in silicon. Phys. Rev. B, 40(8):5484, 1989.

29. A. De Vita, M. J. Gillan, J. S. Lin, M. C. Payne, I. Štich, and L. J. Clarke. Defect Energetics in MgO Treated by First-Principles Methods. Phys. Rev. B, 46(20):12964, 1992.

30. C. Freyria-Fava, R. Dovesi, V. R. Saunders, M. Leslie, and C. Roetti. Ca and Be Substitution in Bulk MgO: Ab Initio HartreeFock and Ionic Model Supercell Calculations. J. Phys. Cond. Matter, 5(27):4793, 1993.

31. L. Giordano, A. Del Vitto, G. Pacchioni, and A. M. Ferrari. CO Adsorption on Rh, Pd and Ag Atoms Deposited on the MgO Surface: a comparative Ab Initio Study. Solid State Sci., 2:161-179, 2000.

32. G. Pacchioni, A. M. Ferrari, and P. Bagus. Cluster and Band Structure $A b$ Initio Calculations on the Adsorption of CO on Acid Sites of the $\mathrm{TiO}_{2}$ (110) Surface. Surf. Sci., 350:159-175, 1995.

33. M. Hellstrom, D. Spangberg, and K. Hermansson. Treatment of Delocalized Electron Transfer in Periodic andEmbedded Cluster DFT Calculations: The Case of $\mathrm{Cu}$ onZnO (10-10). J. Comput. Chem., 36:2394-2405, 2015.

34. P. Persson and M. J. Lundqvist. Calculated Structural and Electronic Interactions of the Ruthenium Dye N3 with a Titanium Dioxide Nanocrystal. J. Phys. Chem., 109:11918-11924, 2005.

35. B. Civalleri, P. Ugliengo, and E. Garrone. Cagelike Clusters as Models for the Isolated Hydroxyls of Silica: Ab-Initio B3LYP Calculations of the Interaction with Ammonia. Langmuir, 15:5829-5835, 1999. 
36. A. M. Ferrari, K. M. Neyman, M. Mayer, M. Staufer, B. C. Gates, and N. Rösch. Faujasite-Supported $\mathrm{Ir}_{4}$ Clusters: A Density Functional Model Study of Metal-ZeoliteInteractions. J. Phys. Chem. $B, 479: 5311-5319,1999$.

37. A. M. Ferrari, R. Soave, A. D'Ercole, C. Pisani, E. Giamello, and G. Pacchioni. Theoretical Characterization of Charge-Transfer Reactions Between $\mathrm{N}_{2}$ and $\mathrm{O}_{2}$ Molecules and Paramagnetic Oxygen Vacancies on the MgO Surface. Surf. Sci., 479:83-97, 2001.

38. A. M. Ferrari and G. Pacchioni. Size and Shape Dependence of the Electrostatic Potential in Cluster Models of the $\mathrm{MgO}$ (100) Surface. Int. J. Quantum Chem., 58:241-250, 1996.

39. J. Baima, A. Zelferino, P. Olivero, A. Erba, and R. Dovesi. Raman Spectroscopic Features of the Neutral Vacancy in Diamond from $\mathrm{Ab}$ Initio Quantum-mechanical Calculations. Phys. Chem. Chem. Phys., 18(3):1961-1968, 2016.

40. A. Zelferino, S. Salustro, J. Baima, V. Lacivita, R. Orlando, and R. Dovesi. The Electronic States of the Neutral Vacancy in Diamond; a Quantum Mechanical Approach. Theor. Chem. Acc., 135(3):1-11, 2016.

41. S. Salustro, A. Erba, C. M. Zicovich-Wilson, Y. Nöel, L. Maschio, and R. Dovesi. Infrared and Raman Spectroscopic Features of the Self-Interstitial Defect in Diamond from Exact-Exchange Hybrid DFT Calculations. Phys. Chem. Chem. Phys., 2016.

42. R. Orlando, R. Dovesi, P. Azavant, N. M. Harrison, and V. R. Saunders. A Super-Cell Approach for the Study of Localized Defects in Solids: Carbon Substitution in Bulk Silicon. J. Phys. Cond. Matter, 6(41):8573, 1994.

43. R. Dovesi, R. Orlando, A. Erba, C. M. Zicovich-Wilson, B. Civalleri, S. Casassa, L. Maschio, M. Ferrabone, M. De La Pierre, P. D'Arco, Y. Noël, M. Causà, M. Rérat, and B. Kirtman. CRYSTAL14: A Program for the Ab Initio Investigation of Crystalline Solids. Int. J. Quantum Chem., 114:1287, 2014.

44. R. Dovesi, V. R. Saunders, C. Roetti, R. Orlando, C. M. ZicovichWilson, F. Pascale, B. Civalleri, K. Doll, N. M. Harrison, I. J. Bush, Ph. D'Arco, and M. Llunell. CRYSTAL 2014 User's Manual. University of Torino, Torino, 2013.

45. A. D. Becke. Density-Functional Thermochemistry. III. The Role of Exact Exchange. J. Chem. Phys., 98(7):5648-5652, 1993.

46. C. Lee, W. Yang, and R. G. Parr. Development of the ColleSalvetti Correlation-Energy Formula into a Functional of the Electron Density. Phys. Rev. B, 37(2):785, 1988.

47. J. S. Binkley, J. A. Pople, and W. J. Hehre. Self-Consistent Molecular Orbital Methods. 21. Small Split-Valence Basis Sets for FirstRow Elements. J. Am. Chem. Soc., 102(3):939-947, 1980.

48. W. J. Hehre, R. F. Stewart, and J. A. Pople. Self-Consistent Molecular-Orbital Methods. I. Use of Gaussian Expansions of Slater-Type Atomic Orbitals. J. Chem. Phys., 51(6):2657-2664, 1969.

49. R. Dovesi, C. Pisani, C. Roetti, and V. R. Saunders. Treatment of Coulomb Interactions in Hartree-Fock Calculations of Periodic Systems. Phys. Rev. B, 28:5781-5792, 1983.

50. M. Causà, R. Dovesi, R. Orlando, C. Pisani, and V. R. Saunders. Treatment of the Exchange Interactions in Hartree-Fock LCAO Calculations of Periodic Systems. J. Phys. Chem., 92:909-913, 1988.

51. F. Pascale, C. M. Zicovich-Wilson, F. Lòpez Gejo, B. Civalleri, R. Orlando, and R. Dovesi. The Calculation of the Vibrational Frequencies of the Crystalline Compounds and its Implementation in the CRYSTAL Code. J. Comput. Chem., 25(6):888-897, 2004.

52. C. M. Zicovich-Wilson, F. Pascale, C. Roetti, V. R. Saunders, R. Orlando, and R. Dovesi. Calculation of the Vibration Frequencies of $\alpha$-Quartz: The Effect of Hamiltonian and Basis Set. $J$. Comput. Chem., 25(15):1873-1881, 2004.

53. A. Erba, M. Ferrabone, R. Orlando, and R. Dovesi. Accurate Dynamical Structure Factors from Ab Initio Lattice Dynamics: the Case of Crystalline Silicon. J. Comput. Chem., 34:346-354, 2013.
54. C. Carteret, M. De La Pierre, M. Dossot, F. Pascale, A. Erba, and R. Dovesi. The Vibrational Spectrum of $\mathrm{CaCO}_{3}$ Aragonite: a Combined Experimental and Quantum-Mechanical Investigation. J. Chem. Phys., 138(1):014201, 2013.

55. J. Baima, M. Ferrabone, R. Orlando, A. Erba, and R. Dovesi. Thermodynamics and Phonon Dispersion of Pyrope and Grossular Silicate Garnets from Ab initio Simulations. Phys. Chem. Minerals, 43:137-149, 2016.

56. L. Maschio, B. Kirtman, R. Orlando, and M. Rérat. Ab Initio Analytical Infrared Intensities for Periodic Systems Through a Coupled Perturbed Hartree-Fock/Kohn-Sham Method. J. Chem. Phys., 137(20):204113, 2012.

57. L. Maschio, B. Kirtman, M. Rérat, R. Orlando, and R. Dovesi. Ab Initio Analytical Raman Intensities for Periodic Systems Through a Coupled Perturbed Hartree-Fock/Kohn-Sham Method in an Atomic Orbital Basis. II. Validation and Comparison with Experiments. J. Chem. Phys., 139(16):164102, 2013.

58. L. Maschio, B. Kirtman, M. Rérat, R. Orlando, and R. Dovesi. Comment on "Ab Initio Analytical Infrared Intensities for Periodic Systems through a Coupled Perturbed Hartree-Fock/Kohn-Sham method" [J. Chem. Phys. 137, 204113 (2012)]. J. Chem. Phys., 139:167101, 2013.

59. M. Ferrero, M. Rérat, R. Orlando, and R. Dovesi. The calculation of static polarizabilities of periodic compounds. the implementation in the crystal code for $1 \mathrm{~d}, 2 \mathrm{~d}$ and $3 \mathrm{~d}$ systems. J. Comput. Chem., 29:1450-1459, 2008.

60. M. Ferrero, M. Rérat, R. Orlando, and R. Dovesi. Coupled perturbed hartree-fock for periodic systems: The role of symmetry and related computational aspects. J. Chem. Phys., 128:014110, 2008.

61. L. Pauling. The Nature of the Chemical Bond. IV. The Energy of Single Bonds and the Relative Electronegativity of Atoms. J. Am. Chem. Soc., 54:3570-3582, 1932.

62. A. Battiato, E. Bernardi, F. Picollo, F. Bosia, M. Lorusso, D. Ugues, A. Zelferino, A. Damin, J. Baima, N. M. Pugno, E. P Ambrosio, and P. Olivero. Softening the Ultra-Stiff: Controlled Variation of Young's Modulus in Single-Crystal Diamond. Acta Mater., 116:95-103, 2016. 